\title{
radoucissement sans formation de surfaces de glissement
}

\author{
stain softening without slip surface
}

\author{
P. HABIB
}

Laboratoire de mécanique des solides ${ }^{2}$

Rev. Franç. Géotech. n` 44, p.p. $5 \cdot 11$ (juillet 1988)

\section{Résumé}

II existe quelques matériaux où la présence d'un maximum de la courbe efforts-déformations n'est pas associée à la formation d'une surface de glissement : roches et bétons fragiles, sables lâches saturés, premier maximum de l'acier doux. L'influence de la fissuration des roches est particulièrement examinée.

\section{Abstract \\ The softening of some materials is not associated with slip surface formation : brittle rocks and concrete, saturated soft sands, first peak of mild steel. Rocks fissures influence is specially examinated.}


Dans plusieurs articles antérieurs ([1], [2], [3] la formation de surfaces de glissement et ses conséquences en mécanique des sols et en mécanique des roches a été examinée en liaison avec la présence d'un maximum de la courbe effort-déformation suivi d'un radoucissement, c'est-à-dire d'une décroissance de la résistance jusqu'au palier appelé parfois " résistance résiduelle».

Si l'on ne connait pas de cas où la formation d'une surface de glissement au cours d'un essai de compression simple ou triaxiale ne soit pas associée à la présence d'un maximum de résistance, il existe par contre quelques cas où la présence d'un maximum de résistance et du radoucissement qui le suit riest pas associée à l'apparition d'une surface de glissement. II est intéressant de chercher à en définir les mécanismes.

\section{LES MATÉRIAUX FRAGILES}

Il est bien connu que lorsqu'on écrase en compression simple des roches fragiles ou des bétons il apparaît, aux bases des cubes ou des éprouvettes cylindriques, des surfaces coniques qui donnent à la rupture une forme en diabolo (fig. 1). Ces surfaces ont souvent été

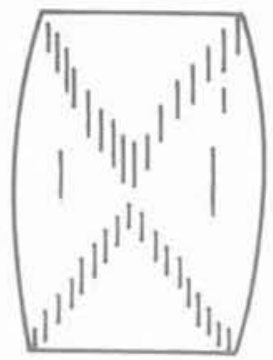

Fig. 1. - Rupture en diabolo et fissuration d'une éprouvette d'un matériau fragile avec frettage par le frottement des faces d'appuis.

Fig. 1. - Diabolo shape failure and fissures in brittle material test piece with confining by friction on the faces in contact with the loading plates.

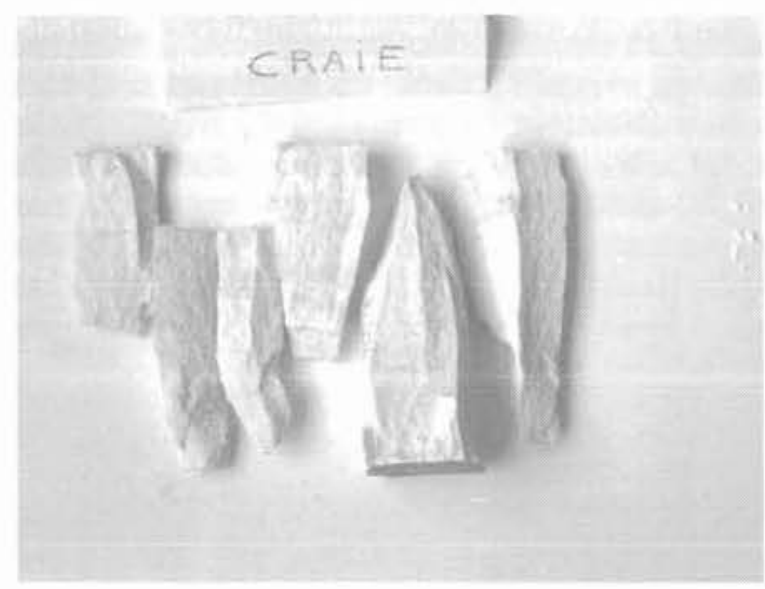

prises pour l'équivalent d'une surface de glissement ou d'une bande de cisaillement, alors qu'en fait elles sont liées au frettage des faces d'appui et qu'elles disparaissent lorsqu'on utilise des dispositifs d'anti-frettage efficaces (figures 2.a, 2.b, 2.c, 2.d, 2.e).
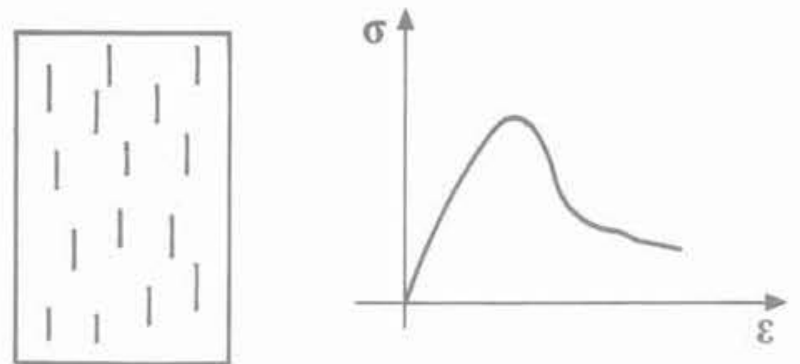

Fig. 2.a - Fissuration d'une éprouvette anti-frettée d'un matériau fragile et courbe de comportement.

Fig. 2.a - Fissures in a brittle material test piece without friction on the loading plates and stress-strain curve assciated.

Avec ou sans diabolos, la rupture est précédée d'une série de crissements correspondant à l'apparition de nouvelles fissures dans le matériau, et ces fissures sont très nombreuses si l'on en juge par la quantité d'émissions acoustiques élémentaires (un pulse = une fissure) qui accompagnent le chargement jusqu'à la rupture complète de l'éprouvette. Ces fissures sont distribuées dans tout le volume et correspondent à des hétérogé. néités de la matière; elles naissent ou s'orientent dans le sens de la contrainte majeure; elles peuvent naître ou se produire dans un joint de grains ou être intracristallines, ou apparaitre au sommet haut et bas d'un trou circulaire, ou au milieu d'un agrégat, ou en se branchant sur une fissure existante (fig. 3). Elles divisent la matière en colonnettes plus ou moins régulières et sont de plus en plus nombreuses lorsque le chargement augmente. Il n'y a pas de localisation particuliere des fissures dans l'éprouvette quand le champ de contrainte est homogène. La courbe effort-déformation en

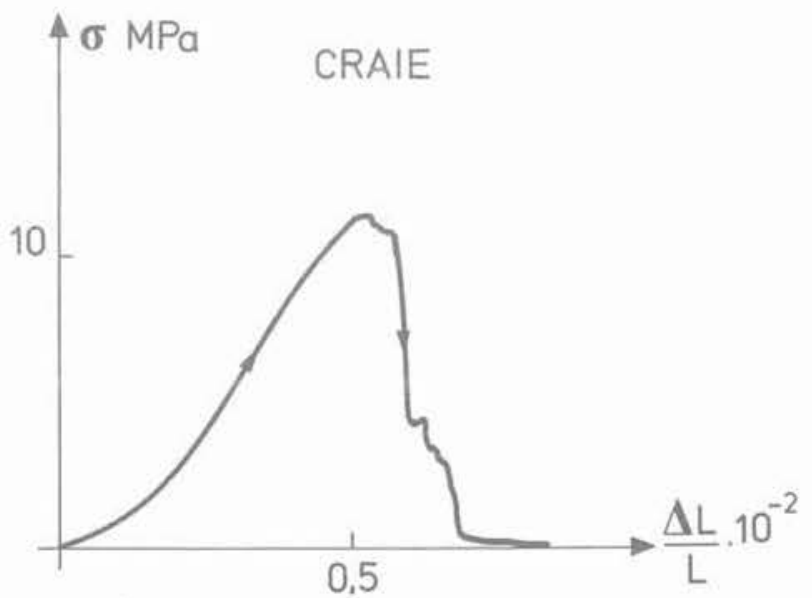

Fig. 2.b: Compression simple anti-frettée d'une craie.

Fig. 2.b: Simple compression without friction on the loading plate: chalk. 

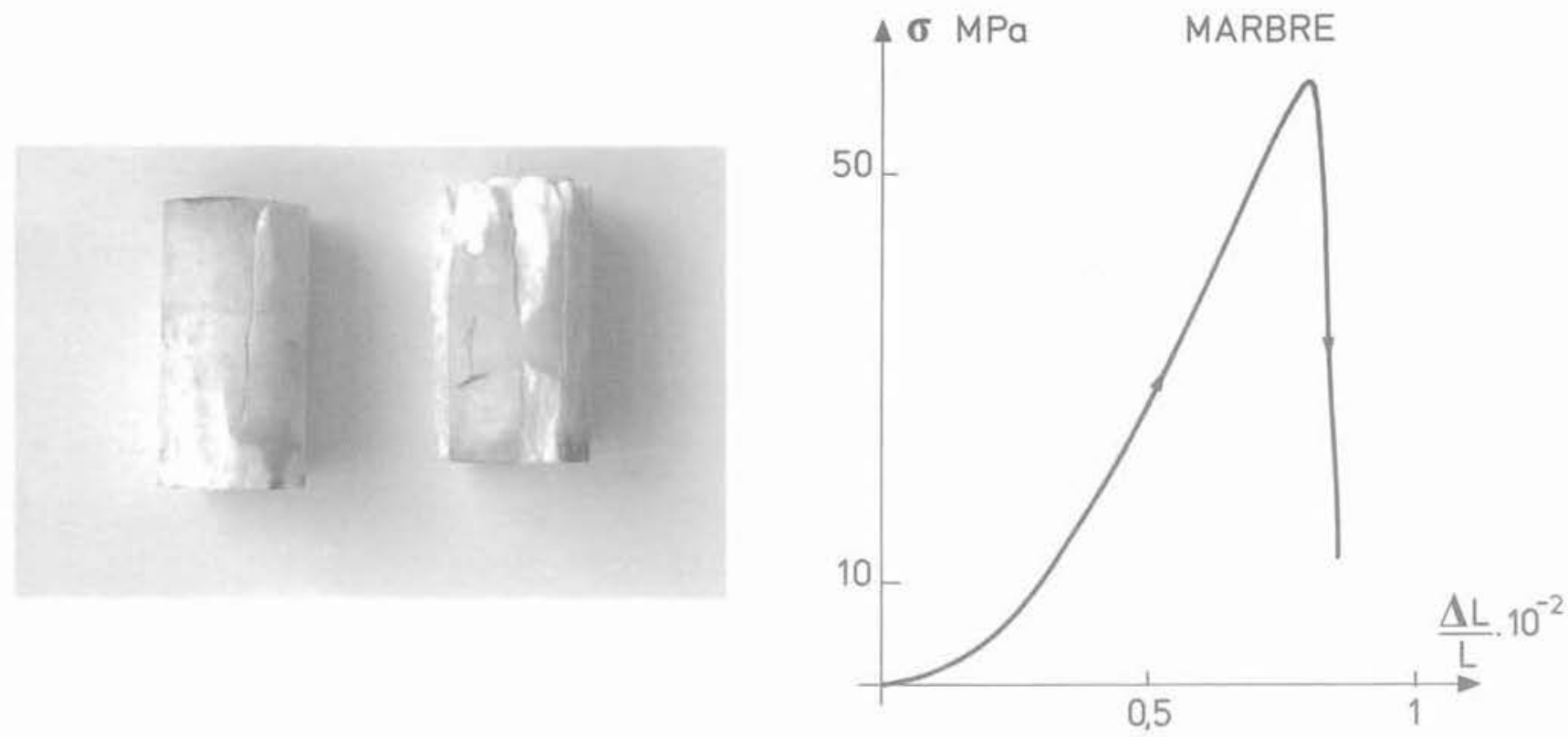

Fig. 2.c: Compression simple anti-frettée d'un marbre. Fig. 2.c : Simple compression without friction on the loading plate: marble.
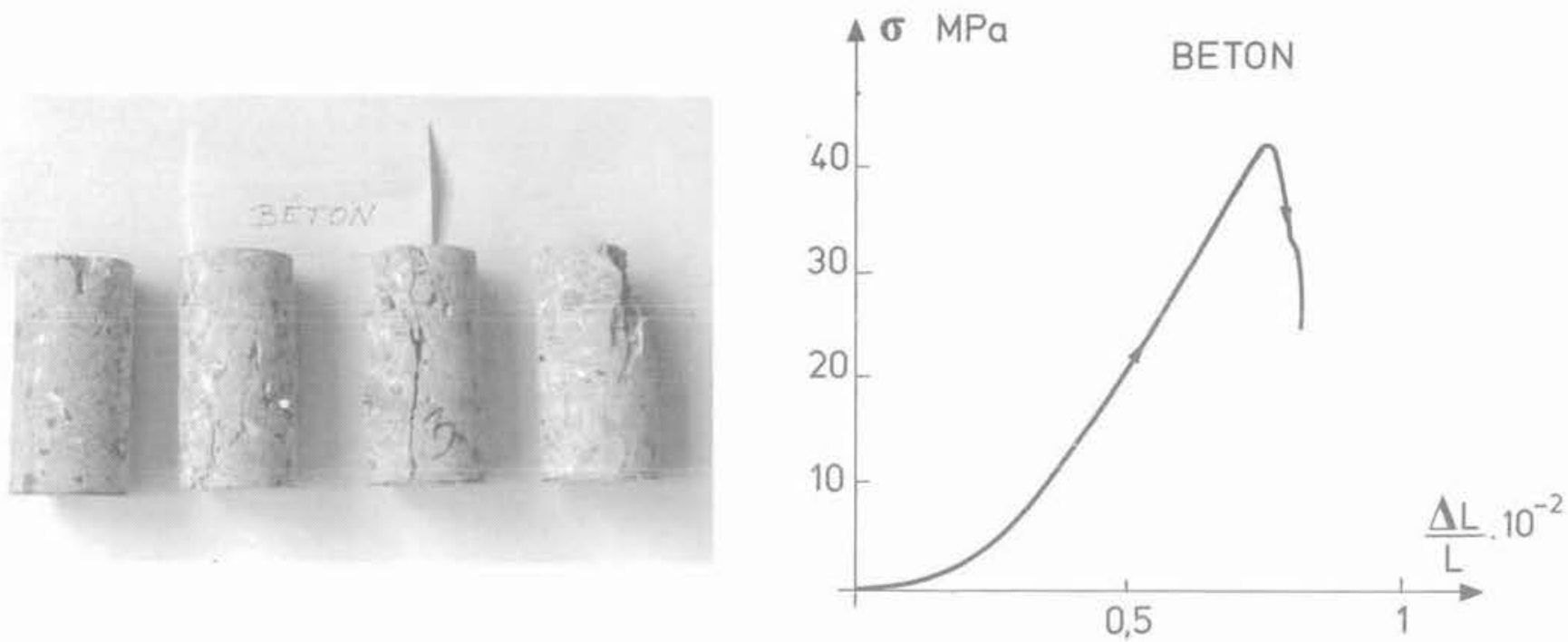

Fig. 2.d: Compression simple anti-frettée d'un béton.

Fig. 2.d: Simple compression without friction on the loa-

ding plate: concrete.

compression simple (fig. 2) présente néanmoins un maximum de résistance, suivi d'un radoucissement au cours d'un essai à déformation commandée, et il n'y a pas formation d'une surface de glissement ou d'une bande de cisaillement.

Il est bien évident que le critère caractérisant l'apparition des premières fissures, ou celui qui caractérise la généralisation des fissures avant la rupture en colonnettes, n'a aucune raison d'être en relation avec le critère caractérisant la rupture en cisaillement avec apparition d'une surface de glissement dont on peut penser qu'il est du type MOHR-COULOMB, même si la courbe intrinsèque n'est pas une droite. On a parlé parfois de rupture en extension pour caractériser de tels modes de destruction. Il y a évidemment là un abus de langage. En effet, l'extension en soi n'a aucune raison d'engendrer une rupture avec séparation. Le gonflement transversal élastique d'une éprouvette de caoutchouc en compression simple, dû à l'effet de POISSON, ni la déformation de contraction transversale dans l'étirement 

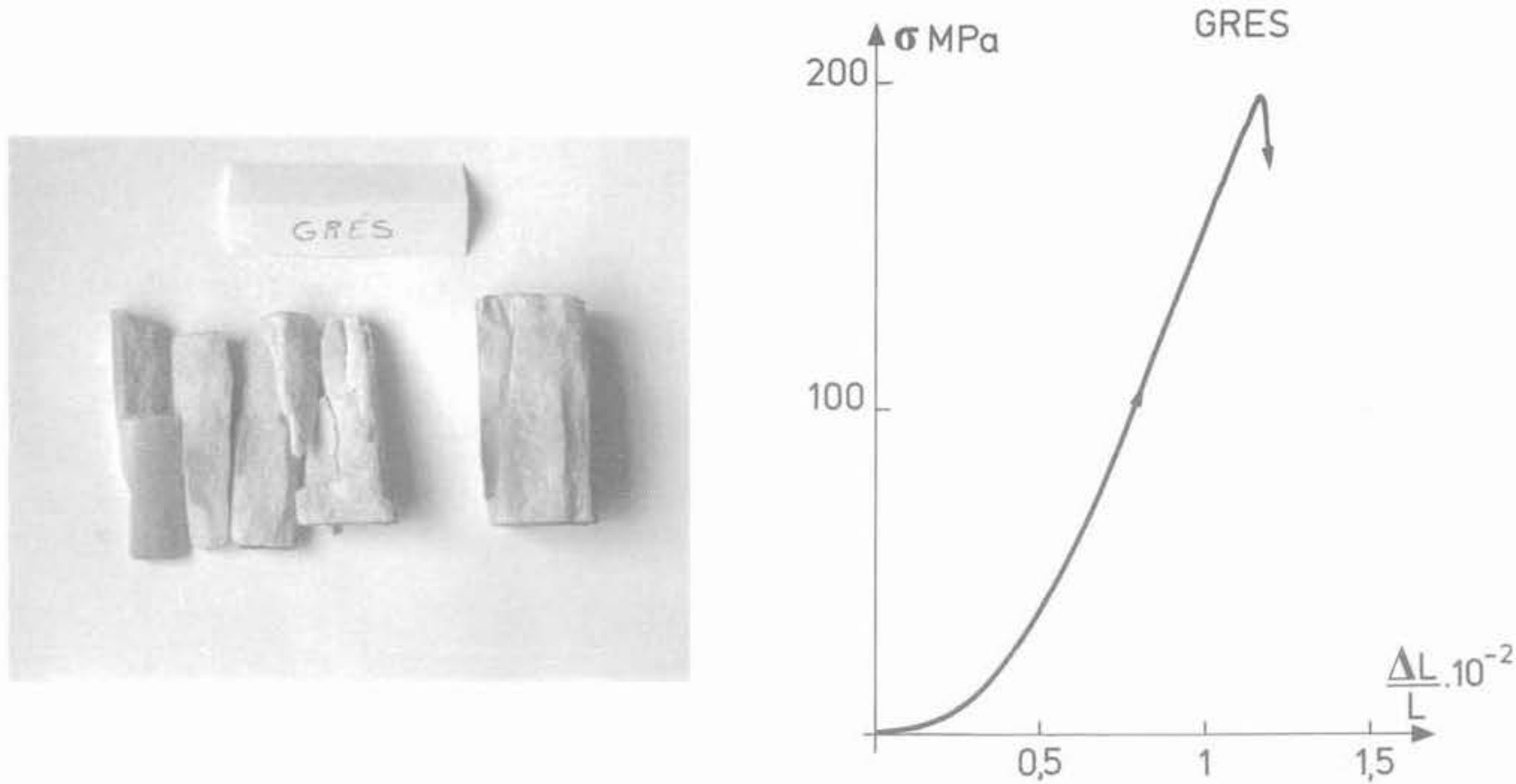

Fig. 2.e: Compression simple anti-frettée d'un grès. Fig. 2.e: Simple compression without friction on the loading plate: sandstone.

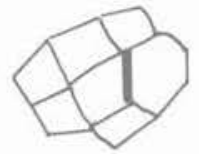

(a)

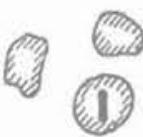

(d)

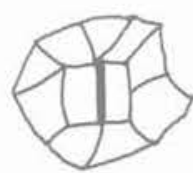

(b)

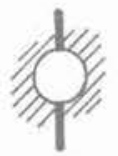

(c)

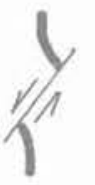

(e)
Mais, dans la rupture en colonnettes des matériaux fragiles, il existe bien des tractions: les craquements que l'on entend à la naissance des fissures, puis l'ouverture de ces fissures montrent bien que la matière a cédé en certains points sous l'effet d'une traction qui se libère par l'ouverture de la fissure. Les endroits où existaient ces tensions sont évidemment associés aux hétérogénéités de la roche ou du béton, hétérogénéités que l'on peut imaginer d'ailleurs aussi bien comme des hétérogénéités physiques (grains différents ou différem. ment orientés) que comme un champ des contrainte résiduelle hétérogène dans un corps homogène.

Plaçons-nous donc juste avant l'apparition des premières fissures. Si l'on coupe fictivement l'éprouvette par un plan parallèle à la contrainte de compression simple, c'est-à-dire par un plan passant par certaines futures fissures, la force normale totale sur la coupure doit être nulle; s'il existe localement des tractions, il faut qu'existent ailleurs des compressions. En définitive, la contrainte normale à ce plan doit ressembler au dessin de la figure 4 (a), mais comme la définition microscopique du milieu nous échappe, pour le moment, il n'est pas possible de raisonner autrement que qualitativement. A lhétérogénéité visible de la matière, se superpose donc un champ de contraintes normales aux facettes parallèles à la contrainte majeure qui est hétérogène. Il est tout à fait vraisemblable que les contraintes tangentielles sur les même facettes soient aussi hétérogènes et, d'une façon générale, que le champ des contraintes sur toutes les facettes soit hétérogène. Lorsque le chargement augmente, la courbe de la figure 4 (a) se déforme par affinité (sauf pour la partie due à des contraintes résiduelles) tant qu'on reste dans le domaine élastique. La naissance d'une fissure provoque localement l'annulation de la contrainte normale de traction (fig. 4(b)) avec redistribution des contraintes à son voisinage immédiat, ce qui permet un plastique d'un fil, qui correspondent l'un à une élongation lautre à un acourcissement transversal ne provoquent de rupture avec séparation. D'une façon générale, les critères de rupture sont exprimés en contraintes, et les rares tentatives (L'HERMITTE, BRICE, etc.) pour exprimer les critères en déformation sont restées sans lendemain, car beaucoup trop artificielles. 


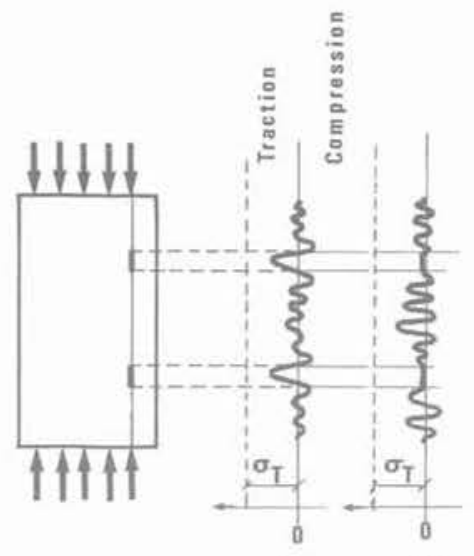

(a) (b)

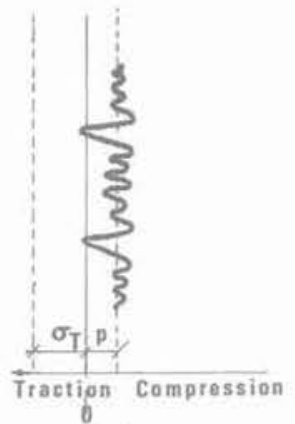

(c)
Fig. 4. - Distribution des contraintes normales sur une facette parallèle à la contrainte majeure avant et après l'apparition des premières fissures.

a et b: Compression simple,

c: Essai avec pression de confinement $p$.

Fig. 4. - Normal stress distribution on a plane parallel to the maximum stress before and after the birth of the first fissures

$a$ and $b$ : Simple compression.

c: Test with a confining pressure $p$

certain nivellement des pointes extrêmes de traction. Si maintenant on superpose à la sollicitation de compression simple une certaine pression $\sigma_{2}=\sigma_{3}$ de confine. ment (essai de type triaxial) le mécanisme est clair. En première approximation, on peut dire que cette pression fait apparaître une contrainte normale moyenne sur la facette envisagée plus haut, à laquelle se superposent les fluctuations des contraintes normales précédentes. L'effet de la pression de confinement est donc de diminuer les pointes de traction, ce qui retarde l'apparition des fissures. Plus le confinement est élevé, plus les fissures sont empêchées de se former. Il existe un niveau de contrainte de confinement tel que toutes les fissures sont a pincées $\nsim$ et ne peuvent plus apparaître ou, tout au moins, ne plus apparaitre avant que d'autres mécanismes de destruction n'apparaissent et en particulier le glissernent ou la distorsion: c'est le phénomène de la transition fragile-ductile.

On remarquera que la division d'une éprouvette d'un matériau homogène en colonnettes verticales n'altère ni son module d'élasticité ni sa résistance, tant que les colonnettes ne flambent pas. Un modèle ainsi constitué doit faire intervenir une hétérogénéité de résistance (ou de déformabilité) des colonnettes élémentaires, de façon que les plus faibles d'entre elles cassent les premières, pour expliquer quelques particularités de la rupture fragile, comme l'effet d'échelle ou la dispersion des résultats. Le fait que la rupture généralisée ne se produise pas dès la première rupture élémentaire montre que le modèle « en série „ (la chaîne qui casse dès la défaillance de son maillon le plus faible) ne convient pas. Le modèle de colonnettes fragiles « en parallèle " avec des résistances différentes permet d'expliquer la non linéarité du comportement et la dégradation du module d'élasticité (fig. 5), mais il est bien clair que ce modèle représente mal le radoucissement et qu'un modèle plus compliqué, par exemple avec des colonnettes de résistances, de longueurs et d'élasticités inégales, c'est-à-dire inégalement résistantes et inégalement chargées, serait préférable. De toutes façons, les modèles à colonnettes en parallèle ne peuvent pas représenter, après le maximum de la force, une résis. tance résiduelle différente de zéro comme cela se produit au cours d'un essai triaxial, ne serait-ce qu'à cause des frottements entre les particules issues de la destruction totale de l'éprouvette.
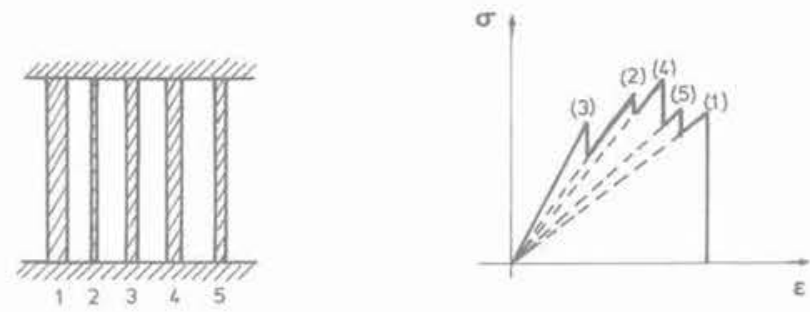

Fig. 5. - Modèle des colonnettes de résistances inégales en parallèle (en déplacement commandé).

Fig. 5. - Model with columns different strengthes (in parallel) (strain controled test)

Si la division en colonnettes verticales ne modifie pas, ou modifie peu, la déformabilité longitudinale, elle augmente par contre beaucoup la déformabilité transversale. II est bien connu que l'apparition des premières fissures dans la direction de $\sigma_{1}$ se traduit par une nette augmentation de la vitesse de déformation transversale $\varepsilon_{3}$. Ce phénomène permet de comprendre la généralisation de la rupture et labsence de localisation.

Lorsqu'un certain volume élémentaire commence à entrer en rupture, il se produit un gonflement transversal qui tend à se transmettre aux volumes adjacents situés en-dessous et au-dessus de lui ; ceux-ci imposent donc au volume qui vient d'entrer en rupture une sorte de frettage qui le renforce, mais, réciproquement, ils subissent une traction transversale (ou une diminution de la contrainte apportée par le confinement) qui les affaiblit et les fait entrer à leur tour en rupture. Ce phénomène est à rapprocher de celui qui a été décrit par TEMPLIER et FINE [6]: un pilier chargé en compression simple constitué dans sa moitié inférieure par une roche dure et raide, dans sa moitié supérieure par une roche déformable beaucoup moins résistante, périt par rupture en traction horizontale dans la roche la plus dure, traction engendrée par la déformation transversale de la roche la plus faible. On peut dire aussi que ce phénomène est l'inverse de celui du frettage par le frottement des faces d'appui d'une éprouvette qui empêche la rupture de se produire dans les cônes d'extrémité (fig. 1). Un phénomène analogue est celui du "boudinage ", rupture en traction d'une inclusion linéaire raide dans une matrice minérale plus molle comprimée perpendiculairement à l'axe de l'inclusion.

Enfin, pour les volumes qui sont latéralement adjacents au domaine qui entre en ruine, la propagation de la rupture suit un mécanisme très simple; la diminution de la résistance du domaine qui se radoucit provoque une redistribution des contraintes dans l'éprouvette, ce qui engendre la surcharge des domaines intacts latéraux jusqu'à leur ruine. En somme, la généralisation de la rupture provient du fait que le début de la rupture par 
fissuration correspond à une rupture dans un élément de volume et non sur un élément de surface, comme cela se produit dans le cas de la rupture par glissement.

\section{LES SOLS EFFONDRABLES}

Les milieux pulvérulents dont la densité relative est de l'ordre de $\mathrm{D}_{t}=20 \%$, lorsqu'ils sont saturés, peuvent présenter un phénomène d'effondrement au cours du chargement. Il s'agit d'une deuxième famille de matériaux qui présentent du radoucissement et une rupture généralisée, c'est-à-dire sans localisation.

Dans ce cas, le mécanisme de la rupture généralisée et du radoucissement est simple. La désorganisation de la structure lâche du sable provoque un transfert des contraintes intergranulaires vers la pression interstitielle. Du fait de l'hétérogénéité de la composition de la structure, il existe probablement un point où cette désorganisation est la plus grande et où la pression interstitielle est maximale et atteint la pression de confinement; la pression interstitielle se transmet alors par continuité dans tout l'échantillon et la résistance intergranulaire s'effondre partout: en effet, si la contrainte totale $\sigma_{3}=\sigma_{3}+\mathrm{u}$ est constante et que $u$ croît jusqu'à atteindre $\sigma_{3}$, alors $\bar{\sigma}_{3}$ tend vers zéro, cependant que $\bar{\sigma}_{1}=\bar{j}_{3}$ tend aussi vers zéro: il y a radoucissement et la déformation est homogène.

Les circonstances où la densité relative est très faible créent une situation de fatigue statique. Le Professeur SEED a réalisé ainsi une expérience particulièrement élégante. Un sable lâche saturé est placé dans un état de contraintes triaxiales tel que le rapport des contraintes principales corresponde à un état déviatorique nettement inférieur à la rupture. Si, en conditions non drainées, on exerce une sollicitation déviatorique brève, comme un choc, il s'initie un fluage de l'échantillon, d'autant plus rapide que le rapport des contraintes principales est proche de la rupture, fluage qui après un délai plus ou moins long (fonction de la vitesse de fluage) aboutit à une perte complète de résistance avec augmentation de la pression interstitielle. Le phénomène peut être arrêté en permettant le drainage de l'éprouvette. Mais, si on reste en conditions non drainées, la rupture est générale et se produit sans formation de surface de glissement.

On peut avoir quelque chose d'analogue avec de la neige poudreuse, le fluide interstitiel étant de l'air et non de l'eau : c'est un des mécanismes des avalanches de neige où le solide se transforme partout en fluide sans quiil y ait de déformation localisée.

\section{L'ACIER}

Sur la courbe de comportement d'un essai de traction d'un acier doux, on distingue classiquement deux maximums de résistance (fig. 6). Le premier qui a lallure d'un petit pic se situe juste avant le palier plastique et le second se situe après la fin de l'écrouissage. La

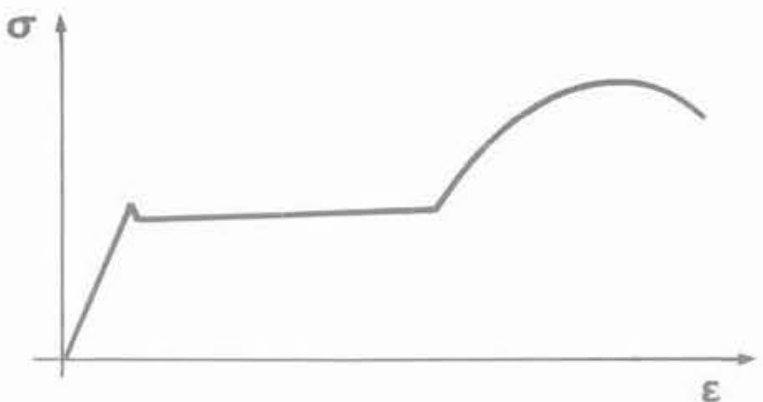

Fig. 6. - Comportement de l'acier doux en traction. Fig. 6. - Mild steel traction test.

formation des lignes de LUDERS (ou de HARTMANN, ou de PIOBERT) est associée au premier pic de la courbe de comportement. Mais, les lignes de LUDERS ne sont pas des lignes de glissement; ce sont simplement des zones où une déformation d'allongement s'est localisée et ces zones s'élargissent par une croissance issue de leur bordure, croissance qui les mène à envahir toute l'éprouvette. On observe des phénomènes analogues dans P'étirement de certains films en matière plastique. Pour les aciers, ce n'est qu'ultérieurement, lorsqu'on franchit le deuxième maximum, que peuvent apparaître des surfaces de glissement nettement identifiées. Il est certain que les deux maximums des courbes de comportement sont très nettement différents. Le premier correspond à un petit pic sur la courbe de comportement et le travail correspondant à l'aire excédentaire est certainement insignifiant, alors que le second donne une courbe de comportement comparable au radoucissement d'autres matériaux. Quoi qu'il en soit, la formation d'une surface de glissement n'est ici associée qu'à lun de ces maximums.

\section{CONCLUSION}

Le cas de l'acier doux avec deux maximums extrême. ment différenciés est évidemment tout à fait particulier. Dans les deux autres exemples par contre, le danger de ruine est diffus dans l'ensemble du volume de la matrice et l'endommagement est réparti dans le matériau, de sorte que le radoucissement peut se produire partout. Dans le cas des roches, les points où les ruptures par traction vont se produire sont inscrits dans la structure hétérogène même si nous ne savons pas déterminer ces emplacements avec précision : il y a des défauts un peu partout, de sorte que les irréversibilités que constituent la création ou l'extension des fissures se produisent dans tout le volume, et comme il ne s'agit pas d'une rupture en cisaillement il n'y a pas de localisation. C'est un peu la même chose pour les sables très lâches: la rupture locale engendre une pression interstitielle qui s'étend à tout l'échantillon et du même coup l'effondrement est général. Pour les lignes de LUDERS, on peut dire que le premier pic de résistance n'est pas non plus associé à une rupture en cisaillement et ceci est peut-être un dénominateur commun à ces différents cas. 


\section{BIBLIOGRAPHIE}

HABIB P. (1984), Les surfaces de glissement en mé. canique des sols. R.F.G., $\mathrm{n}^{\circ} 27$.

HABIB P. (1985), Effet d'échelle et surface de glissement. R.F.G., $n^{\circ} 31$,

HABIB P. (1986), Conditions de formations des surfaces de glissement, R.F.G., $n^{\circ} 34$.
L'HERMITTE R, communication personnelle.

BRICE L.P. (1954), Etude des conditions de formation des fissures de glissement et de décohésion dans les solides. Travaux (juin 1954).

TEMPLIER A. et FINE J. (1969), L'effet de la forme et de l'hétérogénéité des échantillons sur leur résistance en compression. Revue de l'Industrie Minérale, vol. $51, \mathrm{n}^{\circ} 4$, avril 1969. 